\title{
Speech Characteristics and Intelligibility in Adults with Mild and Moderate Intellectual Disabilities
}

\author{
Marjolein C. Coppens-Hofman ${ }^{a}$ Hayo Terband ${ }^{\mathrm{a}-c}$ Ad F.M. Snik ${ }^{d}$ \\ Ben A.M. Maassen ${ }^{c}$ \\ a Department of Medical Psychology and NCEBP, Radboud University Nijmegen Medical Centre, Nijmegen, \\ bUtrecht Institute of Linguistics OTS, Utrecht University, Utrecht, 'Centre for Language and Cognition (CLCG), \\ University of Groningen, Groningen, and d Department of Otorhinolaryngology and Donders Institute for Brain, \\ Cognition and Behaviour, Radboud University Nijmegen Medical Centre, Nijmegen, The Netherlands
}

\section{Keywords}

Speech · Intelligibility · Disabilities and handicaps ·

Perceptual analysis $\cdot$ Intellectual disabilities $\cdot$ Speech

disorders · Apraxia $\cdot$ Phonological disorders

\section{Abstract}

Purpose: Adults with intellectual disabilities (ID) often show reduced speech intelligibility, which affects their social interaction skills. This study aims to establish the main predictors of this reduced intelligibility in order to ultimately optimise management. Method: Spontaneous speech and picture naming tasks were recorded in 36 adults with mild or moderate ID. Twenty-five naïve listeners rated the intelligibility of the spontaneous speech samples. Performance on the picture-naming task was analysed by means of a phonological error analysis based on expert transcriptions. Results: The transcription analyses showed that the phonemic and syllabic inventories of the speakers were complete. However, multiple errors at the phonemic and syllabic level were found. The frequencies of specific types of errors were related to intelligibility and quality ratings. Conclusions: The development of the phonemic and syllabic repertoire ap-

\begin{tabular}{|c|c|}
\hline KARGER & $\begin{array}{l}\text { (c) } 2017 \text { The Author(s) } \\
\text { Published by S. Karger AG, Basel } \\
1021-7762 / 17 / 0684-0175 \$ 39.50 / 0\end{array}$ \\
\hline www.karger.com/fpl & $\begin{array}{l}\text { This article is licensed under the Creative Commons Attribution- } \\
\text { NonCommercial-NoDerivatives } 4.0 \text { International License (CC BY- } \\
\text { NC-ND) (http://www.karger.com/Services/OpenAccessLicense). } \\
\text { Usage and distribution for commercial purposes as well as any dis- } \\
\text { tribution of modified material requires written permission. }\end{array}$ \\
\hline
\end{tabular}

pears to be completed in adults with mild-to-moderate ID. The charted speech difficulties can be interpreted to indicate speech motor control and planning difficulties. These findings may aid the development of diagnostic tests and speech therapies aimed at improving speech intelligibility in this specific group.

(c) 2017 The Author(s)

Published by S. Karger AG, Basel

\section{Introduction}

Communication is an important aspect of quality of life, and speech is the primary means of human communication. In adults with intellectual disabilities (ID), speech communication is often troubled by disordered speech production and/or impaired hearing [1], resulting in miscommunication and consequently impairing social interactions, possibly behavioural problems and isolation.

Speech intelligibility can be defined as how clearly a person speaks so that his or her speech is comprehensible to a listener [2]. Reduced speech intelligibility leads to misunderstanding, frustration, and loss of interest by 
communication partners. As a result, communication decreases or remains at a low level. Thus, to improve the quality of life of adults with ID, it is essential to enable them to make themselves understood. Impairment of speech production is among the most commonly reported difficulties in children, adolescents and adults with ID [3]. The deficiencies are not resolved when growing up and speech intelligibility remains a problem throughout life [4]. Yet, still little is known about the predictors of the reduced speech intelligibility in adults with ID.

\section{Speech Intelligibility}

Speech intelligibility is usually approached as word or utterance recognition in natural communication situations [5]. The intelligibility of a spoken message is influenced by a number of factors. Above all, intelligibility is a joint product of the speaker and the listener. Listener factors are familiarity with the speaker and with the topic of conversation [6,7]. Familiarity with the speaker is especially helpful when speech is disordered or poorly intelligible due to speech errors $[6,7]$. In addition, intelligibility varies with the nature of the spoken material (e.g., linguistic structure and length of utterance) and the context of communication (e.g., the quality of the acoustic transmission of the speech signal, the availability of visual cues from the speaker, and contextual support for the message to be transmitted). A gold standard for speech intelligibility measurement is not available, but it can be argued that a standardised speech intelligibility assessment can be obtained by making audio-recordings of spontaneous speech samples in an interview situation where the proband is asked to tell about neutral, everyday situations. These audio-recordings are presented to naïve listeners, who either produce transcriptions or give intelligibility judgments (for an overview of methods of standardisation, see [7]).

\section{Speech Difficulties in Adults with ID}

Speech characteristics have not been studied widely in adults with ID in general, but Roberts et al. [1] documented the following characteristics of the speech of adults with Down syndrome: consonant cluster reduction; final consonant deletion; unstressed syllable omission, mostly at the start of a multisyllabic word; and consonant substitution (e.g., a fricative sound /s/ becomes a plosive / $\mathrm{t} /$ ). Errors in the speech of persons with Down syndrome have been characterised to be inconsistent $[1,8,9]$, and the production errors and articulation difficulties observed mainly occur in the phonemes that are typically acquired in the final stages of normal speech-language development [10-12]. Van Borsel attributed the errors to a developmental delay [10]. Unfortunately, no data are available for adults with ID of mixed aetiology or other groups of persons with a specific ID.

The present study set out to examine the common difficulties in the speech of adults with ID of mixed aetiology, thereby focusing on the main predictors of the reduced intelligibility of their speech. Objectified measures that form a strong predictor of intelligibility may be used to evaluate treatment outcomes, and the observed speech irregularities may become therapeutic targets. To this end, a quantitative analysis of standardised speech samples elicited in a picture naming task was made resulting in inventories of segmental (phonemic) and syllabic error characteristics. In addition, intelligibility of spontaneous speech samples was judged by naïve listeners such that the relevance of the quantified speech characteristics in determining reduced speech intelligibility could be assessed. An additional factor to consider as a potential cause of reduced intelligibility is poor auditory feedback due to deficient auditory processing in combination with chronic hearing difficulties [1]. In the current study, we took hearing difficulties into account on a functional level by measuring the auditory discrimination abilities of all our participants, and by analysing any relation with speech difficulties. Finally, severity of ID and aetiology were included in the analysis.

\section{Method}

This study was approved by the Ethics Committee of the Radboud University Nijmegen Medical Centre. Potential participants and their legal representatives received written and oral information on the study, after which oral and written consent was obtained from the legal representatives, parents, caregivers, and participants.

\section{Participants}

Inclusion was based on the level of ID, i.e. IQ 40-70 (DSMIV - Mild and Moderate). The 36 participants that entered the study (19 men and 17 women aged $18-40$ years) had all been identified as poorly intelligible by their caregivers and relatives. Their speech problems had not been assessed before by diagnostic tests and the cause of the reduced intelligibility was unclear. As to ID aetiologies, 11 of the 36 participants had Down syndrome, 1 participant had Fragile-X syndrome, and another Turner syndrome, 3 participants had sustained brain damage in the first year of life, 2 had a chromosomal deficiency (microcephaly), 1 had suffered trauma, and another 2 had suffered hypoxia during birth, while during the study 2 participants were diagnosed with a specific genetic microdeletion [13]; in 12 cases, the cause of the ID was unknown. Sixteen of the participants were classified as having a mild ID (IQ 55-70) and 20 as having a moderate ID (IQ 40-55). For the 
analysis of a possible effect of ID aetiology, the aetiologies with a small $(n<10)$ number of cases were aggregated, thus creating 4 groups (unknown $[n=12]$; Down syndrome $[n=11]$; other syndromes [Fragile X, Turner syndrome, microcephaly, genetic microdeletion; $n=6$ ]; acquired ID [oxygen deficiency, brain damage, electrographic status epilepticus of sleep, trauma; $n=7]$ ).

\section{Data Collection}

Recordings of spontaneous speech production and a picturenaming task were obtained from all participants. To determine the participants' vocabulary level and test their understanding of the instructions, all participants took the Peabody Vocabulary Test [14] 1 week prior to the individual recording sessions. Participants were thus also able to familiarise themselves with the researcher (first author), the recording equipment, and setting.

In testing situations, people with ID often speak in subdued tones or use explosive voice modulations - vocal characteristics which can severely limit the quality of the speech signal in both live-voice and tape-recorded presentations. Special care was therefore given to optimise the speech signals in our samples. Some of the participants indeed tended to speak quietly, while in others vocal intensity varied from utterance to utterance, all prosodic variations that could confound the goals of the assessment to some extent. In these cases, feedback was offered prior to the actual recording to reinforce or modify the participant's speech efforts. Moreover, all speech recordings were made in a quiet, familiar room in the participant's own care or residential facility by the same researcher (first author), who was seated at the same table opposite the participant, allowing eye contact with the participant. Also, a silent observer was present during each recording session, which mostly involved a parent, the primary caregiver or a person close to the participant. In no case was the participant asked to come to an unknown place or a clinical setting.

The first recording contained 3-10 min of spontaneous speech in response to open questions about the participants' hobby's and daily pastime. As it is appropriate to use a closed-set evaluation for people with ID using items within their linguistic competence, a second recording was made using Logo-Art [15]. This Dutch picture-naming test consists of 128 easily recognisable pictures that represent words of everyday life and was developed to assess articulation skills in children aged $4-8$ years. The target words included all vowels, diphthongs, consonants and consonant clusters used in the Dutch language in all word positions (initial, medial, and final).

A professional solid-state recorder (Marantz PMD620) was used to obtain digital speech samples. As several participants found the external microphone threatening or highly distracting, we placed the internal microphone at approximately $40 \mathrm{~cm}$ distance from the speaker's mouth. All recordings were made in the same way using a bit rate of $705 \mathrm{kbps}$ and a frequency of $44.1 \mathrm{kHz}$. The duration of the recordings varied depending on the participant's movements, in-between conversation, pace of responding and speech rate. No participant was rushed at any time. The LogoArt recordings took $20-40 \mathrm{~min}$.

\section{Data Processing}

Spontaneous Speech

Of the 36 participants, 34 produced a sufficient amount of spontaneous speech for an intelligibility judgment. Two participants were excluded from the assessment as they only used oneword utterances, consistently repeating this one word.

Speech Characteristics and Intelligibility in Adults with ID
Two relevant, continuous segments were selected from each of the 34 speech samples by use of the PRAAT software programme [16] based on auditory and visual cues in the recorded speech, yielding a total of 68 segments to be judged. The segments each contained a total of 2 min of uninterrupted sentences or ongoing speech. These were assessed for intelligibility by 25 speech and language pathology students in their final year of training. All students resided in the same socio-geographical environment as the participants. Before the speech assessments, the students listened to 3 random speech samples of non-participants (similarly aged adults with mild or moderate ID) to familiarise themselves with the speech recordings, the rating method and overall setting. All speech samples were presented through loudspeakers at normal loudness in a quiet room. The students were asked to individually rate speech intelligibility on a 5 -point scale, with 1 denoting essentially unintelligible, 3 intelligible at times, and 5 essentially intelligible. The mean scores of the 25 listeners were taken to indicate the intelligibility of a participant's speech.

\section{Picture-Naming Task and Transcription}

Picture-naming performance on the Logo-Art, in terms of the words produced in response to the presented images, was transcribed in broad phonetic alphabet (IPA) and keyed into the Logical International Phonetics Program (LIPP) transcription system [17]. This computer-based transcription system allows transcribed utterances to be analysed with respect to their phonetic characteristics, e.g. by providing an inventory of phonemes and syllable structures, and compared to target utterances, yielding variables such as percentage of consonants correct (PCC) and number of cluster reductions (for details, see below).

The participants recognised and correctly named more than $85 \%$ of the Logo-Art pictures. Utterances produced while the participant was chewing or had fingers or objects in or over the mouth, as well as yells, grunts, and coughs were excluded. All recordings were independently transcribed by 2 professional transcribers (both speech language pathologists) who did not know the participants and were not informed about ID nature and cause. Transcription reliability was assessed by comparing multiple transcriptions of the same utterances. Mean inter-rater segment-by-segment correspondence in all transcriptions was $94 \%$ and higher.

\section{Analysis of Logo-Art Transcripts}

Comparison of produced and target utterances was conducted at the segmental and syllable-structure level. Analyses at the segmental level concerned the identity of the segments and yielded 2 types of variables: proportions of consonants correct (PCC; both overall and separated out for different developmental complexity levels [18]), and proportions of substitutions (overall and in relation to syllable position). The substitutions were further classified as typical or atypical phonological processes [18]. Analyses at the syllabic level concerned the structure of the syllables only, not the identity of the phonemes within the syllable. The syllable structures that were evaluated were: $\mathrm{V}$ (vowel); $\mathrm{CV}$ (consonant-vowel); VC; VCC; CVC; CCVC; CVCC; CCVCC; CCCVC; and CCCVCC. For each of these structures, the percentage correct was computed. The resulting variables are detailed in Table 1.

\section{Statistical Analysis}

Data analysis was layered. First, we explored possible influences of the different between-subject factors, hearing loss, severity of 
ID, and ID aetiology group, on the intelligibility scores as well as the general phonological measures using a series of analyses of variance.

The second step consisted of a more in-depth analysis of the specific types of errors and phonological processes, including an assessment of the different stages in phonological acquisition (according to the Phonological Analysis of Dutch [18]).

Finally, multiple linear regression analyses were carried out to identify which (subset of) the general phonological measures could reliably be used as a predictor of speech intelligibility. The regression analysis featured the stepwise method with backward elimination with a removal criterion of 0.1 based on the $F$ statistic.

Significance level was set at $p<0.05$, while $p$ values $<0.10$ were qualified as trends. Homogeneity of variance was tested with the Levene test of homogeneity, and the Mauchly test of sphericity was applied.

\section{Results}

\section{General Factors}

First, the influence of the different between-subject factors on the main speech parameters was investigated using a series of univariate analyses of variance with the intelligibility scores, as well as the phonological measures (Table 1) as dependent variables, and hearing loss, severity of ID, and ID aetiology group as fixed factors. The analyses yielded a significant effect of severity of ID on intelligibility $(F(1,33)=8.60, p<0.01)$, as well as trend effects for this factor on the proportions syllable structures correct (PSSC; $F(1,33)=3.14, p=0.09)$ and consonant deletion (PCDel; $F(1,33)=3.20, p=0.09$ ). No further effects or interactions were observed. Therefore, the data were collapsed over the factors hearing loss, and ID aetiology.

In a subsequent multivariate analysis of variance on the differences in intelligibility scores and phonological measures, with severity of ID only, this latter factor was significant $(F(12,21)=3.19, p<0.01)$. Mean intelligibility scores were higher in the participants with mild ID (mean 3.32; SD 0.77; range 2.15-4.61) as compared to the moderate group (mean 2.44, SD 0.69; range 1.32-3.60). The outcomes on the general phonological measures are

Table 1. Overview of the variables determined by the segmental and syllable structure comparison of target word and produced utterance

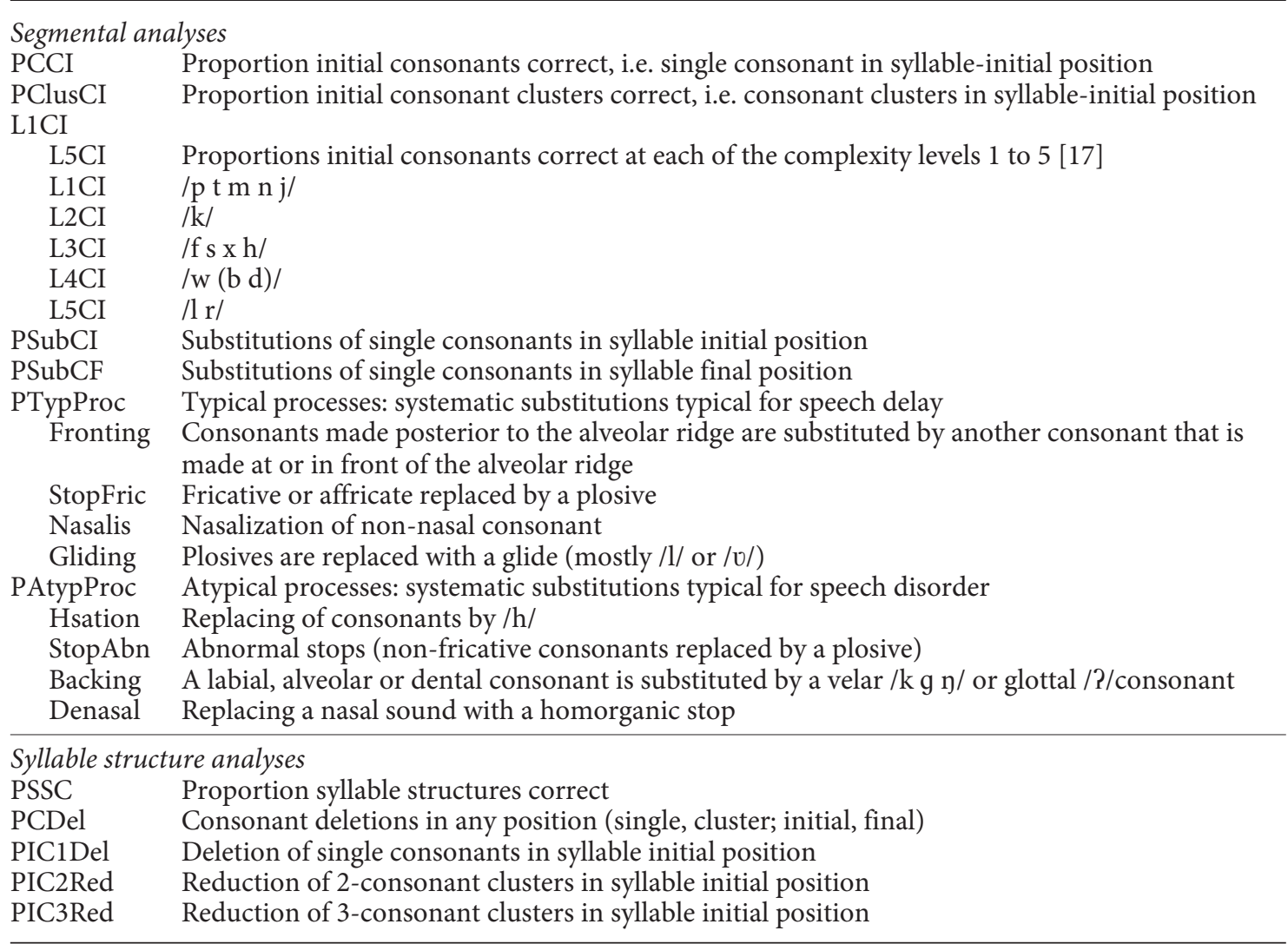


presented in Figures 1 and 2. Univariate analysis showed a trend of a higher proportion of correct consonants in syllable-initial position (PCCI) in the mild ID as compared to the moderate group $(F(1,33)=3.510, p=0.07$; Fig. 1). Furthermore, the participants with moderate ID made significantly more substitutions of single conso-

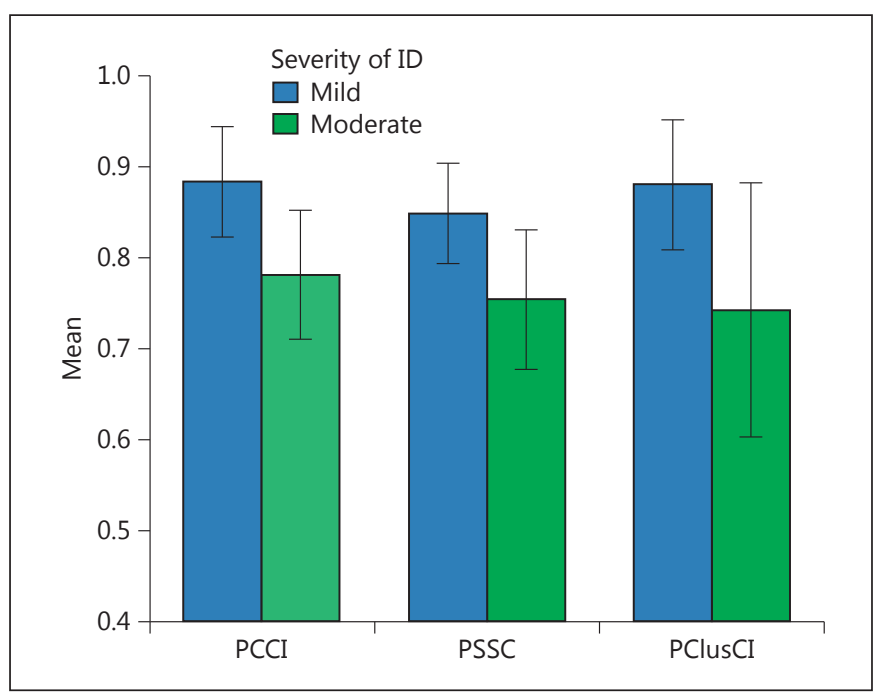

Fig. 1. Mean scores on the 3 measures of phonological accuracy: proportion consonants correct in syllable-initial position (PCCI), proportion syllable structures correct (PSSC), proportion consonant clusters correct in initial position (PClusCI). Error bars indicate $95 \% \mathrm{CI}$. nants in syllable initial position (PSubCI; $F(1,33)=$ $5.097, p<0.05)$ and deletions of single initial consonants $(\mathrm{PIC} 1 \mathrm{Del} ; F(1,33)=5.012, p<0.05)$ than their peers with mild ID (Fig. 2).

The main error types concern the pronunciation of single and clustered initial consonants as reflected by the high frequencies of single consonant deletions and cluster reductions in syllable-initial position. In order to gain more insight into the processes underlying the production difficulties, we conducted further phonological analyses. Our first query was whether the error patterns were similar to the phonological delay observed in otherwise unimpaired children. To this end, we compared the phonemic inventory of our sample with the patterns described for typically developing children during speech acquisition.

\section{Phonemic Inventory}

Beers developed a system to analyse phonological development, called the Phonological Analysis of Dutch [18]. She found that the completeness of the phonemic inventory can be assessed in 5 levels of complexity. An incomplete inventory typically results in a pattern in which the higher levels are produced at a lower percentage accuracy. The speakers with mild ID tended to produce higher proportions consonants correct than the speakers with moderate ID (Table 2). The mean percentages correct for all complexity levels were above $75 \%$, in-
Fig. 2. Mean scores on the 7 phonological error measures: proportion substitutions of single consonants in initial position (PSubCI), proportion substitutions of single consonants in syllable-final position (PSubCF), proportion abnormal substitution processes (PAbnProc; h-sation, abnormal stopping, backing, and denasalisation), proportion normal substitution processes (PNormProc; fronting, stopping of fricatives, nasalisation, and gliding), proportion consonant deletions (PCDel), proportion deletion of consonants in syllableinitial position (PIC1Del), and the proportion reduction of consonant clusters in syllable-initial position containing 2 and 3 consonants (PIC2Red, and PIC3Red). Error bars indicate $95 \% \mathrm{CI}$.

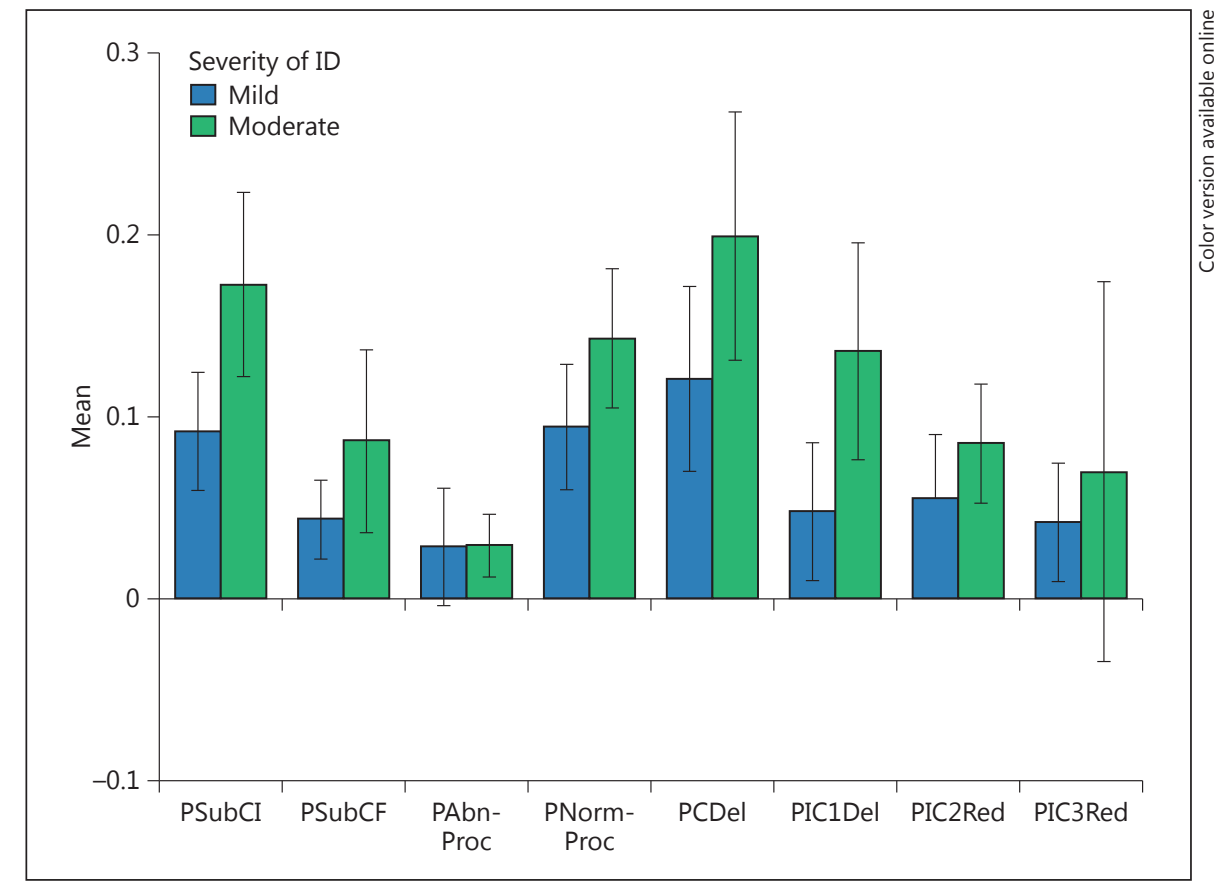

Speech Characteristics and Intelligibility in Adults with ID
Folia Phoniatr Logop 2016;68:175-182 DOI: $10.1159 / 000450548$ 
Table 2. Mean proportions and standard deviations (SD) of correctly produced consonants according to developmental levels of complexity [17]

\begin{tabular}{|c|c|c|c|}
\hline \multirow{2}{*}{$\begin{array}{l}\text { Levels of speech } \\
\text { development }\end{array}$} & \multicolumn{3}{|c|}{ Severity of ID } \\
\hline & $\begin{array}{l}\text { mild } \\
(n=16)\end{array}$ & $\begin{array}{l}\text { moderate } \\
(n=20)\end{array}$ & $\begin{array}{l}\text { total } \\
(n=36)\end{array}$ \\
\hline L1CI & $0.90 \pm 0.11$ & $0.77 \pm 0.21$ & $0.83 \pm 0.18$ \\
\hline $\mathrm{L} 2 \mathrm{CI}$ & $0.93 \pm 0.12$ & $0.85 \pm 0.16$ & $0.88 \pm 0.15$ \\
\hline L3CI & $0.82 \pm 0.18$ & $0.77 \pm 0.17$ & $0.80 \pm 0.17$ \\
\hline L4CI & $0.93 \pm 0.09$ & $0.82 \pm 0.17$ & $0.87 \pm 0.15$ \\
\hline L5CI & $0.89 \pm 0.15$ & $0.75 \pm 0.21$ & $0.81 \pm 0.20$ \\
\hline
\end{tabular}

L1CI-L5CI, level of complexity 1-5 of consonants in syllable initial position.

Table 3. Means and standard deviations (SD) of proportions of atypical phonological processes

\begin{tabular}{lll}
\hline & \multicolumn{2}{l}{ Severity of ID } \\
\cline { 2 - 3 } & $\begin{array}{l}\text { mild } \\
(n=16)\end{array}$ & $\begin{array}{l}\text { moderate } \\
(n=20)\end{array}$ \\
\hline H-Sation & $0.08 \pm 0.15$ & $0.06 \pm 0.08$ \\
StopAbn & $0.01 \pm 0.03$ & $0.02 \pm 0.03$ \\
Backing & $0.02 \pm 0.05$ & $0.04 \pm 0.06$ \\
Denasal & $0.10 \pm 0.19$ & $0.05 \pm 0.12$ \\
\hline
\end{tabular}

dicating that, overall, the phonological repertoire is complete and that no systematic pattern can be discerned that reflects a phonological delay. Those participants who produced lower percentages correct did not show a declining tendency at the higher complexity levels; thus, these participants make errors irrespective of phonological complexity.

\section{Typical and Atypical Speech Processes}

Young children and (young) adults with speech difficulties may produce errors that affect entire classes of sounds rather than individual sounds. At a particular age, these so-called phonological processes (a detailed description is provided in Table 1) are a normal, natural part of their speech development and therefore denoted as typical processes. A speech profile consisting of processes that are typical for younger children can be interpreted as speech delay. Thus, consonant deletion in syllable-final position, fronting, consonant-cluster reduction and stopping are considered typical speech processes.
In contrast, atypical speech processes comprise classes of errors that are not normal during any stage of speech development and are therefore taken to indicate speech pathology. The atypical processes we analysed are: h-sation, abnormal stopping, backing, and denasalisation; all in syllable-initial position. The results are presented in Table 3 (see also Fig. 2). Our analyses revealed 2 dominant atypical speech processes, irrespective of severity of ID. We first found a high proportion of h-sations and, secondly, a high proportion of denasalisations.

To summarise, the number of errors in the speech production of our sample was high. For most participants, the phoneme repertoire appears to be well-completed. However, on average, the participants showed high error rates across complexity levels, and many typical as well as atypical errors in their speech.

\section{Predictors of Intelligibility}

But which of the deviations in the speech of our participants with ID account for its reduced intelligibility? The clinical relevance, of course, is that since phonemic quality contributes to intelligibility, the observed irregularities may become therapeutic targets. Furthermore, phonological error measures that form a strong predictor of intelligibility may be used to evaluate treatment outcomes. Multiple regression analyses (method: stepwise with backward elimination) were conducted with intelligibility - the students' ratings of spontaneous speech as the dependent variable and the phonological error measures of the Logo-Art transcription analysis as the independent variables.

For the mild ID group, the model with PCCI, PIC3Red, PNormProc and PClusCI showed up in the regression analysis $\left(R^{2}=0.790\right.$, adj $R^{2}=0.706$, standard error $=0.42$, $p=0.002$ ). These 4 measures together accounted for $79 \%$ of the variance in intelligibility in this group. For the moderate ID group the model with PCCI, PIC2Red, and PClusCI showed that these 3 measures together were responsible for $69 \%$ of the variance $\left(R^{2}=0.693\right.$, adj $R^{2}=$ 0.631 , standard error $=0.42, p=0.000)$.

\section{Discussion}

The participants with mild and moderate ID of the present study showed distinct difficulties in their speech production that affect both the quality and intelligibility of their verbal output. Their speech is characterised by an overall high error rate and the occurrence of both typical 
and atypical phonological processes; the percentages of correct productions at all levels of phonemic complexity showed a non-declining pattern, indicating deviant speech development rather than an incomplete phonemic inventory. We found no significant difference between our speech parameters based on hearing loss, or ID aetiology. The severity of ID (mild vs. moderate), however, did show a strong association with speech intelligibility and was also related to particular error frequencies.

The phonological processes cannot be explained by weakness or paralysis of the speech muscles or other sensorimotor deficits alone. Kumin [3] documented that symptoms of 'childhood verbal apraxia' could be found in children with Down syndrome. Among the symptoms of apraxia that were identified [3], we observed: inconsistency in phoneme (sound) productions, difficulties in combining and sequencing phonemes (like in consonant clusters), and speech-rhythm problems; that is, as has been reported for Down syndrome, speech patterns in adults with ID show many errors not seen in typical development.

To our knowledge, we are the first to analyse prompted and unprompted speech of adults with various cognitive impairments. The participants were selected on the basis of their reduced speech intelligibility and their wish to improve their verbal communication skills but had never been tested for or diagnosed with any speech disorders. Our results showed the participants to have a complete phonemic and syllabic repertoire and also showed distinctive processes in their speech production. It is worth mentioning that our speech samples were recorded in a quiet room, with a microphone positioned close to the speaker, causing the effects of low speaking volume and other voice characteristics on intelligibility to be relatively small. These are likely to be far more detrimental when communication takes place in a busy and noisy environment with poor acoustics (such as echo resulting from safety and hygiene regulations) that characterises many residential and work facilities for people with ID.

Another issue to consider in this context is that shortterm and long-term verbal memory are both highly involved in speech production and that the 2 systems are impaired in people with ID [19-21]. From a psycholinguistic perspective, a distinction can be made between representations stored in memory and the transformation and execution processes that utilise these representations. Word production and phonological planning skills must first be acquired and then become automated through practice. Short-term memory plays an important role in these ongoing processes of planning, sequenc-

Speech Characteristics and Intelligibility in Adults with ID ing and coordination of speech movements. Long-term memory is essential for the storage of word forms; deficits in long-term memory therefore can account for the persistence of the speech difficulties into adulthood.

Based on our results, we propose that the deviant speech characteristics in this population should be targeted in tailored therapeutic management schemes, and that they can serve as measures to evaluate treatment results. Speech skills can improve through dedicated training under the guidance of speech language pathologists specialised in working with people with cognitive deficits. Continuously providing feedback in understandable terms and offering and demonstrating the right learning processes in well-structured exercises are essential elements in optimising the speech output in people with impaired cognitive functions.

\section{Conclusions}

Naiive ratings of the intelligibility of the spontaneous speech of adults with mild to moderate ID related significantly to the phonemic and syllabic variables derived from expert transcriptions of their verbal output obtained with a picture-naming task. The main speech difficulties in this group of impaired speakers appear to be related to underlying speech motor planning difficulties. There are early indications that tailored speech therapy can remediate some of the speech deficits in adults with ID, fostering their communicative skills by reducing the severity of their speech difficulties.

References

1 Roberts JE, Price J, Malkin C: Language and communication development in Down syndrome. Ment Retard Dev Disabil Res Rev 2007;13:26-35.

2 Leddy M: The biological bases of speech in people with Down syndrome; in Miller J, Leddy M, Leavitt LA (eds): Improving the Communication of People with Down Syndrome. Baltimore, Paul H Brookes Publishing, 1999, pp 61-80.

3 Kumin L: Speech intelligibility and childhood verbal apraxia in children with Down syndrome. Down Syndr Res Pract 2006;10:10-22.

4 Chapman RS, Seung HK, Schwartz SE, Bird EK: Predicting language production in children and adolescents with Down syndrome: the role of comprehension. J Speech Lang Hear Res 2000;43:340-350.
Folia Phoniatr Logop 2016;68:175-182 DOI: $10.1159 / 000450548$
181 
5 Smith LE, Nelson CL: International intelligibility of English: directions and resources. World English 1985;4:333-342.

6 Tjaden K, Liss, JM: The role of listener familiarity in the perception of dysarthric speech. Clin Linguist Phonet 1995;9:139-154.

7 Kent RD: Speech intelligibility and communicative competence in children; in Kaiser AP, Gray DB (eds): Enhancing Children's Communication: Research Foundations for Intervention. Baltimore, Brookes Publishing, 1992, vol 2, pp 223-239.

8 Coppens-Hofman MC, Maassen BAM, van Schrojenstein Lantman-de Valk HMJ, Snik AFM: Speech difficulties and poor speech intelligibility in adults with Down syndrome: a review of the literature. J Hear Sci 2012;2:916.

9 Dodd B, Thompson L: Speech disorder in children with Down's syndrome. J Intellect Disabil Res 2001;45:308-316.
10 van Borsel J: Articulation in down syndrome adolescents and adults. Eur J Disord Commun 1996;31:415-444.

11 Barnes E, Roberts J, Long SH, Martin GE, Berni MC, Mandulak KC, Sideris J: Phonological accuracy and intelligibility in connected speech of boys with fragile X syndrome or Down syndrome. J Speech Lang Hear Res 2009;52:1048-1061.

12 Kent RD, Vorperian HK: Speech impairment in Down syndrome: a review. J Speech Lang Hear Res 2013;56:178-210.

13 Willemsen MH, Vallès A, Kirkels LAMH, Mastebroek M, Olde Loohuis N, Kos A, Wissink-Lindhout WM, de Brouwer APM, Nillesen WM, Pfundt R, Holder-Espinasse M, Vallée L, Andrieux J, Coppens-Hofman MC, Rensen $\mathrm{H}$, Hamel BCJ, van Bokhoven $\mathrm{H}$, Aschrafi A, Kleefstra T: Chromosome 1p21.3 microdeletions comprising DPYD and MIR137 are associated with intellectual disability. J Med Genet 2011;48:810-818.

14 Dunn LH, Dunn LM: Peabody Picture Vocabulary Test-III-NL (PPVT-III-NL) (Schlichting L, transl). Amsterdam, Pearson, 2005.
15 Baarda D, de Boer-Jongsma N, HaasjesJongsma W: Logo-art articulatieonderzoek. Ternat/Axel, Baert, 2005.

16 Boersma P, Weenink D: Praat: Doing phonetics by computer; http://www.praat.org/ (retrieved March 10, 2013).

17 Intelligent Hearing Systems: Logical international phonetics program. 2012; http://www. ihsys.com/site/LIPP.asp?tab=4.

18 Beers M: The Phonology of Normally Developing and Language-Impaired Children. Amsterdam, University of Amsterdam, 1995.

19 Vicari S: Memory development and intellectual disabilities. Acta Paediatr 2004;93:60-63.

20 Jarrold C, Baddeley AD, Phillips CE: Verbal short-term memory in Down syndrome: a problem of memory, audition, or speech? J Speech Lang Hear Res 2002;45:531-544.

21 Brock J, Jarrold C: Serial order reconstruction in Down syndrome: evidence for a selective deficit in verbal short-term memory. J Child Psychol Psychiatry 2005;46:304-316. 